\title{
Precision spectroscopy of pionic atoms and chiral symmetry in nuclei
}

Kenta Itahashi ${ }^{1, \star}$, DeukSoon Ahn ${ }^{1}$, Georg P.A. Berg ${ }^{2}$, Masanori Dozono ${ }^{1}$, Daijiro Etoh ${ }^{3}$, Hiroyuki Fujioka ${ }^{4}$, Naoki Fukuda ${ }^{1}$, Nobuhisa Fukunishi ${ }^{1}$, Hans Geissel ${ }^{5}$, Emma Haettner ${ }^{5}$, Tadashi Hashimoto ${ }^{1}$, Ryugo S. Hayano ${ }^{6}$, Satoru Hirenzaki ${ }^{7}$ Hiroshi Horii ${ }^{6}$, Natsumi Ikeno ${ }^{8}$, Naoto Inabe ${ }^{1}$, Masahiko Iwasaki ${ }^{1}$, Daisuke Kameda ${ }^{1}$, Shouichiro Kawase ${ }^{9}$, Keiichi Kisamori ${ }^{1,9}$, Yu Kiyokawa ${ }^{9}$, Toshiyuki Kubo ${ }^{1}$, Kensuke Kusaka ${ }^{1}$, Masafumi Matsushita ${ }^{9}$, Shin'ichiro Michimasa ${ }^{9}$, Go Mishima ${ }^{6}$, Hiroyuki Miya ${ }^{9}$, Daichi Murai ${ }^{1}$, Hideko Nagahiro ${ }^{7}$, Takahiro Nishi ${ }^{1}$, Shinsuke Ota ${ }^{9}$, Naruhiko Sakamoto ${ }^{1}$, Kimiko Sekiguchi ${ }^{3}$, Hiroshi Suzuki ${ }^{1}$, Ken Suzuki ${ }^{10}$, Motonobu Takaki ${ }^{9}$, Hiroyuki Takeda ${ }^{1}$, Yoshiki K. Tanaka ${ }^{5,6}$, Tomohiro Uesaka ${ }^{1}$, Yasumori Wada ${ }^{3}$, Yuni N. Watanabe ${ }^{6}$, Helmut Weick ${ }^{5}$, Hiroki Yamakami ${ }^{4}$, Yoshiyuki Yanagisawa ${ }^{1}$, and Koichi Yoshida ${ }^{1}$

\footnotetext{
${ }^{1}$ Nishina Center for Accelerator-Based Science, RIKEN, Saitama 351-0198, Japan

2 JINA and Department of Physics, University of Notre Dame, Indiana 46556, USA

${ }^{3}$ Department of Physics, Tohoku University, Sendai 980-8578, Japan

${ }^{4}$ Department of Physics, Kyoto University, Kyoto 606-8502, Japan

${ }^{5} \mathrm{GSI}$ Helmholtzzentrum für Schwerionenforschung GmbH, D-64291 Darmstadt, Germany

${ }^{6}$ Department of Physics, University of Tokyo, Tokyo 113-0033, Japan

${ }^{7}$ Department of Physics, Nara Women's University, Nara 630-8506, Japan

${ }^{8}$ Department of Regional Environment, Tottori University, Tottori 680-8551, Japan

${ }^{9}$ Center for Nuclear Study, University of Tokyo, Tokyo 113-0033, Japan

${ }^{10}$ Stefan Meyer Institute, Austrian Academy of Sciences, A-1090 Vienna, Austria
}

\begin{abstract}
We conduct an experimental project to make spectroscopy of deeply bound pionic atoms systematically over wide range of nuclei. We aim at studying the strong interaction in the low energy region, which has close connection to spontaneous chiral symmetry breaking and its partial restoration in nuclear matter. First experimental results show improved spectral resolution and much better statistical sensitivity than previous experiments. Present status of the experiment is reported.
\end{abstract}

\section{Introduction}

Strong interaction in low-energy region exhibits various interesting aspects. One of the particularly interesting aspects is spontaneous breakdown of chiral symmetry, which is characterised by the order parameter of the symmetry, chiral condensate. Pionic atoms, bound systems of a negatively charged pion to a nucleus, can be used for investigating the chiral symmetry in the nucleus [1-3], where the high density results in partial restoration of the chiral symmetry. Theoretically, interaction strength

^e-mail: itahashi@riken.jp 
between pion and nucleus has a close connection with the partial restoration $[4,5]$, and precision determination of the isovector interaction sets quantitative constraints to the chiral condensate $\langle\bar{q} q\rangle$ in the nuclear medium.

Orbitals of pions in "deeply bound pionic atoms" are largely overlapping with the nuclei, and the pionic levels and widths reflect the $s$-wave pion-nucleus interaction $[2,3]$, which is phenomenologically formulated in the Ericson-Ericson optical potential [6]. The $s$-wave part of the optical potential is described in the following form;

$$
\begin{aligned}
-2 \mu U_{s-\text { wave }}(r) & =4 \pi \epsilon_{1}\left\{b_{0} \rho(r)+b_{1} \Delta \rho(r)\right\}+4 \pi \epsilon_{2} B_{0} \rho^{2}(r), \\
\rho(r) & =\rho^{(\mathrm{n})}(r)+\rho^{(\mathrm{p})}(r), \\
\Delta \rho(r) & =\rho^{(\mathrm{n})}(r)-\rho^{(\mathrm{p})}(r),
\end{aligned}
$$

where $\epsilon_{1}=1+\mu / M=1.147, \epsilon_{2}=1+\mu / 2 M=1.073$ with $M$ being the nucleon mass and $\mu$ being the reduced mass of the pion. $b_{0}, b_{1}$ and $B_{0}$ are the $s$-wave potential parameters. $\rho^{(n)}(r)$ and $\rho^{(p)}(r)$ are radial neutron and proton density distributions of a nucleus, respectively.

Among the parameters to parametrize the potential, isovector parameter $b_{1}$ is known to have closeconnection with the chiral condensate at a finite density through the Gell-Mann-Oaks-Renner relation [7] and Tomozawa-Weinberg relation [8, 9]. Systematic measurement of pionic tin isotopes was performed in GSI, and the energy levels and widths of $1 s$ states in the pionic tin 115, 119, and 123 isotopes were measured [1]. The measured energy levels and widths were analyzed to deduce the $b_{1}$ parameter in medium leading to quantitative evaluation of $\langle\bar{q} q\rangle$ in nuclear medium to be reduced by $30 \%$ compared with that in vacuum although the deduced value was associated with relatively large errors. In order to deduce the chiral condensate with better precision, high precision measurement of pionic atoms was awaited.

\section{Experiment}

Aiming at unprecedented spectral resolution of $300 \mathrm{keV}$ (FWHM), we conducted an experimental spectroscopy of pionic tin isotopes at RI Beam Factory, RIKEN, Japan [10], using $\left(d,{ }^{3} \mathrm{He}\right)$ nuclear reaction for the production of the pionic atoms as the method of the reaction spectroscopy was established in the former experiments [11-14]. The Q-value of the reaction was precisely measured to deduce the mass of the reaction product, pionic atoms, in the missing mass spectroscopy. The produced pionic atoms are observed as peak structures in the Q-value spectrum or in the excitation spectrum of the reaction product below the free pion emission threshold. In the $\left(d,{ }^{3} \mathrm{He}\right)$ nuclear reaction, a neutron is picked up from the target nucleus hence the produced pionic bound states are coupling with a neutron hole state in the core nucleus.

We employed $500 \mathrm{MeV}$ deuteron beam accelerated by the Superconducting Ring Cyclotron (SRC). A remarkable characteristics of the RI Beam Factory used as "Pionic Atom Factory (piAF)" is its high current primary beam with an intensity of $\geq 10^{12} / \mathrm{s}$, which helps measurement with a good statistical sensitivity. As for the targets, we chose ${ }^{122} \mathrm{Sn}$ and ${ }^{117} \mathrm{Sn}$ and placed them at the nominal target position. There are theoretical calculations for the production cross section of pionic atoms in the $\left(d,{ }^{3} \mathrm{He}\right)$ reactions [15-17]. An isotope with an odd neutron number was chosen for the first time. We expected good separation of spectral strengths with valence neutron pick-up from those with coreneutron pick-up [18]. The emitted ${ }^{3} \mathrm{He}$ in the $\left(d,{ }^{3} \mathrm{He}\right)$ reaction were momentum analyzed by BigRIPS projectile fragment separator used as a forward spectrometer with the momentum resolving power of $\delta p / p \sim 3000$. 


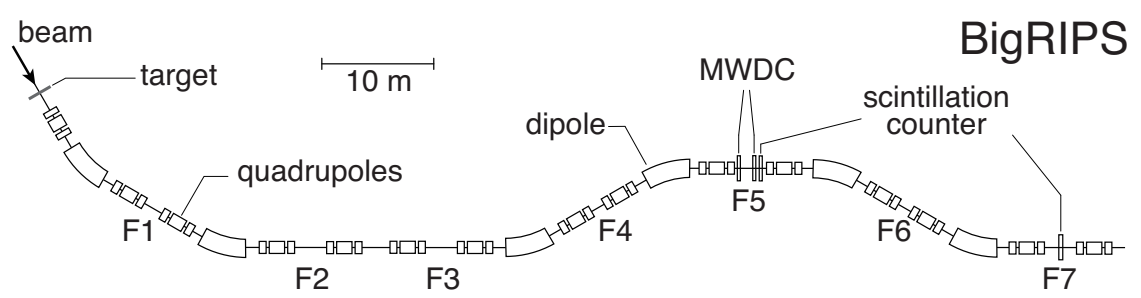

Figure 1. Experimental setup at BigRIPS. The $500 \mathrm{MeV}$ deuteron beam was extracted from SRC impinging on the target. We installed two sets of MWDCs at F5 dispersive focal plane and sets of scintillation counters at F5 and F7.

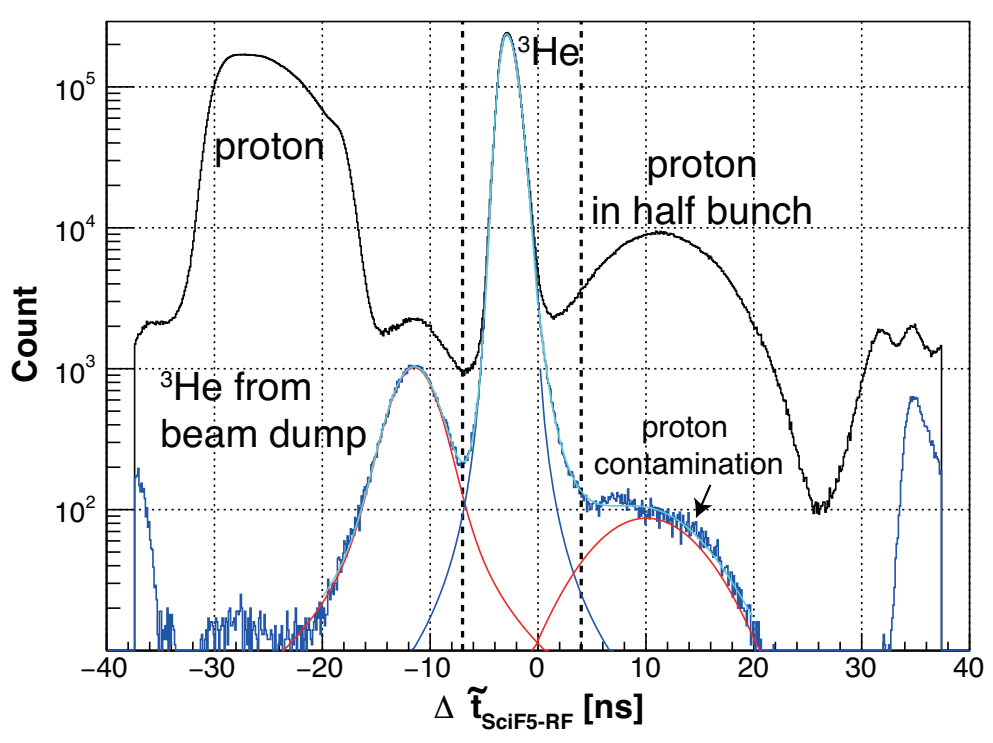

Figure 2. Measured relative timing of particles at F5 to RF phase. The central peak corresponds to ${ }^{3} \mathrm{He}$. A cut condition has been applied to select ${ }^{3} \mathrm{He}$. Proton contamination level is estimated to be $\leq 10^{-3}$ after combining with other cut conditions.

Figure 1 schematically depicts the experimental setup. We placed two sets of multi-wire drift chambers (MWDCs) at the dispersive focal plane (F5) and measured the ${ }^{3} \mathrm{He}$ tracks for the momentum analysis. We also placed a segmented scintillation counter at F5 and a scintillation counter at an achromatic focal plane F7 to identify the particles by the energy loss in the scintillation counters, by the time of flight between F5 and F7, and by relative timing to the radio frequency (RF) of SRC.

Figure 2 shows a measured relative timing spectrum of particles at F5 to the RF phase. Since the particles impinging on the target are bunched and synchronized to the RF phase, the relative timing reflects the time-of-flight from the target to F5. The central peak corresponds to ${ }^{3} \mathrm{He}$. We applied a cut condition indicated by the vertical dashed lines to select ${ }^{3} \mathrm{He}$. Proton contamination level is controlled to be $\leq 10^{-3}$ after combining with other cut conditions. 


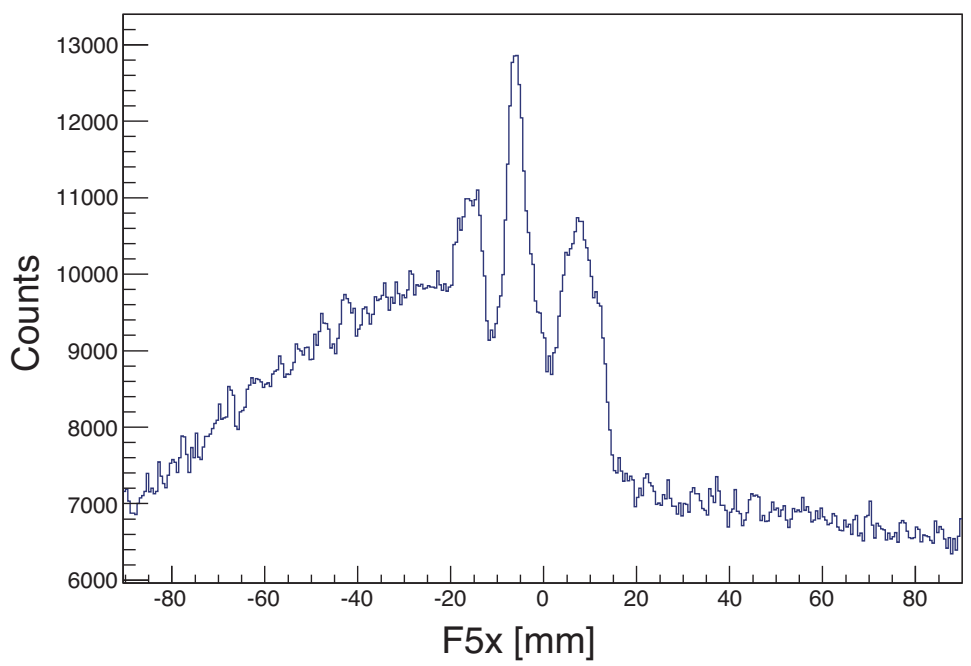

Figure 3. Measured acceptance uncorrected position spectrum at F5. Right side corresponds to lower excitation energy of the reaction products. Nearly linear background is observed in the region $20-80 \mathrm{~mm}$, which is due to nuclear excitation without pion production. At left side $-80--30 \mathrm{~mm}$, we observe quasifree pion production continuum. Peak structures near the center corresponds to production of pionic atoms. The right-most peak is due to $1 s$ pionic atom formation coupling with a neutron hole state of $3 s_{1 / 2}$. The central peak is mainly due to pionic $2 p$ states.

Figure 3 shows a typical acceptance-uncorrected position spectrum measured at F5. The right side of the spectrum corresponds to higher ${ }^{3} \mathrm{He}$ momentum. Spectral strengths are standing on a nearly linear background of nuclear excitation unassociated with pion production. The peaks near the center of the spectrum are originating in the formation of pionic atoms, and the right-most peak is associated with the pionic $1 s$ state coupling mainly with $3 s_{1 / 2}$ neutron hole state. The achieved experimental resolution is estimated to be as good as $\sim 300 \mathrm{keV}$ (FWHM). Further analysis to improve the experimental resolution and to deduce the binding energies and widths is ongoing.

\section{Conclusion}

The present piAF project makes full use of the advantages of the facility and aims at performing systematic high precision spectroscopy of pionic atoms. Recent experiments achieved unprecedented resolution and precision of the measurement by optimization of the experimental conditions including partial application of the dispersion-matching ion-optics. A simultaneous measurement of $1 s$ and $2 p$ pionic levels will also contribute to reduction of the systematic uncertainties in the relative energy scale. Considering possible perturbative effects of pionic orbitals to nuclei, systematic study of pionic atoms over wide range of nuclei will also benefit in comprehensive understanding of the strong interaction and the nuclear structure.

\section{Acknowledgments}

This work is supported by MEXT Grants-in-Aid for Scientific Research on Innovative Areas (Nos. JP22105517, JP24105712 and JP15H00844). 


\section{References}

[1] K. Suzuki et al., Phys. Rev. Lett. 92, 072302 (2004)

[2] R. S. Hayano and T. Hatsuda, Rev. Mod. Phys. 82, 2949 (2010)

[3] T. Yamazaki et al., Phys. Rept. 514, 1 (2012)

[4] E. E. Kolomeitsev, N. Kaiser, and W. Weise, Phys. Rev. Lett. 90, 092501 (2003)

[5] D. Jido, T. Hatsuda and T. Kunihiro, Phys. Lett. B 670, 109 (2008)

[6] M. Ericson and T. E. O. Ericson, Ann. Phys. 36, 323 (1966)

[7] M. Gell-Mann, R. J. Oakes, and B. Renner, Phys. Rev. 175, 2195 (1968)

[8] Y. Tomozawa, Nuovo Cimento A 46, 707 (1966)

[9] S. Weinberg, Phys. Rev. Lett. 17, 616 (1966)

[10] Y. Yano, Nucl. Instr. Meth. Phys. Res. B 261, 1009 (2007)

[11] T. Yamazaki et al., Z. Phys. A 355, 219 (1996)

[12] H. Gilg et al., Phys. Rev. C 62, 025201 (2000)

[13] K. Itahashi et al., Phys. Rev. C 62, 025202 (2000)

[14] H. Geissel et al., Phys. Rev. Lett. 88, 122301 (2002)

[15] N. Ikeno et al., Prog. Theor. Phys. 126, 483 (2011)

[16] N. Ikeno, H. Nagahiro and S. Hirenzaki, Euro. Phys. J. A 47, 161 (2011)

[17] N. Ikeno et al., Prog. Theor. Exp. Phys. 2015, 033D01 (2015)

[18] N. Ikeno et al., Prog. Theor. Exp. Phys. 2013, 063D01 (2013) 